\title{
Prognostic Significance of Serum CEA for Non-small Cell Lung Cancer Patients Receiving Stereotactic Body Radiotherapy
}

\author{
TAKASHI SHINTANI, YUKINORI MATSUO, YUSUKE IIZUKA, TAKAMASA MITSUYOSHI, \\ TAKASHI MIZOWAKI and MASAHIRO HIRAOKA \\ Department of Radiation Oncology and Image-applied Therapy, \\ Graduate School of Medicine, Kyoto University, Kyoto, Japan
}

\begin{abstract}
Background/Aim: To examine the prognostic significance of serum carcinoembryonic antigen (CEA) for stage I non-small cell lung cancer (NSCLC) treated with stereotactic body radiotherapy (SBRT). Patients and Methods: In total, 129 stage I NSCLC patients were analyzed and divided into two groups: CEA-High (CEA>5 $\mathrm{ng} / \mathrm{ml})$ and CEA-Low (CEA $\leq 5 \mathrm{ng} / \mathrm{ml})$. Results: Median follow-up time was 38 months. Overall survival was not significantly different between CEA-High $(n=47)$ and CEA-Low $(n=82)$ patients $(57 \%$ vs. $63 \%$ at 3 years; $p=0.39)$, although progression-free survival (PFS) was significantly worse in CEA-High patients $(31 \%$ vs. $51 \%$ at 3 years; $p=0.01)$. Larger tumor size and high CEA level were independent prognostic factors for worse PFS. Failure pattern analysis showed that regional node or distant recurrence was more common in CEA-High patients (47\%) than in CEA-Low patients (29\%). Conclusion: Patients with CEA-High stage I NSCLC have a higher risk of regional or systemic relapse and should be followed-up carefully.
\end{abstract}

Stereotactic body radiotherapy (SBRT) for non-small cell lung cancer (NSCLC) achieves high local control with limited toxicity $(1,2)$ and is regarded as standard treatment for inoperable stage I NSCLC $(3,4)$. Phase III randomized controlled trials of surgery versus SBRT in operable patients were discontinued as a result of poor accrual, and it is therefore unclear whether SBRT is comparable to surgery for such patients (5). However, a retrospective study has reported

Correspondence to: Yukinori Matsuo, Department of Radiation Oncology and Image-applied Therapy, Graduate School of Medicine, Kyoto University, 54 Shogoin Kawahara-cho, Sakyo-ku, Kyoto, 606-8507, Japan. Tel: +81 757513762, Fax: +81 757719749, e-mail: ymatsuo@kuhp.kyoto-u.ac.jp

Key Words: Carcinoembryonic antigen (CEA), non-small cell lung cancer (NSCLC), stereotactic body radiotherapy (SBRT). excellent outcomes of SBRT in operable patients (6), and the number of patients treated with SBRT because of unwillingness to undergo surgery is increasing. Compared with surgery, SBRT is less invasive and is well tolerated. A disadvantage of SBRT, however, is the lack of accurate pathological staging (particularly for regional node metastasis), which might negatively affect oncological outcomes.

Serum carcinoembryonic antigen (CEA) is an established tumor marker for NSCLC, although modest elevation of CEA is often seen without malignancy (7). According to retrospective studies, high CEA level was a negative prognostic factor for survival and a risk factor for occult regional node metastasis in clinical stage I NSCLC patients undergoing surgery (8). Several factors, such as tumor size, sex, and solid tumor, are reported to be prognostic factors, but the prognostic impact of CEA level for stage I NSCLC patients treated with SBRT has not been reported to date (9, 10). Therefore, we conducted this study to determine whether prognosis and failure pattern differ according to CEA level.

\section{Materials and Methods}

Patients and study design. Patient eligibility criteria were as follows: (1) pathologically confirmed NSCLC; (2) clinical T1a-2aNOM0 (Union for International Cancer Control staging criteria, 7th edition); (3) SBRT treatment between January 2005 and December 2013 for curative intent; and (4) pretreatment serum CEA level available. Exclusion criteria were as follows: (1) synchronous or metachronous cancer within 5 years of SBRT treatment; (2) insufficient follow-up data (less than 6 months' follow-up without death or recurrence); and (3) neoadjuvant or adjuvant systemic therapy. Patients were staged with computed tomography (CT) of the chest and upper abdomen and brain magnetic resonance imaging (MRI) (or brain CT if there were contraindications to MRI, such as implanted cardiac pacemakers). ${ }^{18}$ F-fluorodeoxyglucose-positron emission tomography (FDG/PET) was typically used for staging at our center. Clinical staging, operability, and choice of treatment were discussed at a multidisciplinary tumor board consisting of radiation oncologists, respiratory surgeons, medical oncologists, and diagnostic radiologists. Serum CEA was measured by electrochemiluminescence immunoassay (ECLISA) (Roche Diagnostics, Rotkreuz, Switzerland). 
The upper limit of CEA was $5 \mathrm{ng} / \mathrm{ml}$, according to the manufacturer. This retrospective study was approved by our institutional review board.

SBRT procedure. The details of the SBRT procedure at our institution have been published previously (11). Patients were immobilized with Stereotactic Body Frame (Elekta, Stockholm, Sweden) before March 2008 and with BodyFIX (Elekta, Stockholm, Sweden) thereafter. The treatment machine used was Clinac2300 C/D (Varian Medical Systems, Palo Alto, CA, USA) before April 2008, Novalis system (BrainLAB, Feldkirchen, Germany) from April 2008 to November 2010, and Vero4DRT (Mitsubishi Heavy Industries, Ltd. Hiroshima, Japan) after November 2010. The treatment plan was made on Eclipse (Varian Medical Systems, Palo Alto, CA, USA) using pencil beam convolution with heterogeneity correction of Batho power law until June 2009 and iPlan (BrainLAB, Feldkirchen, Germany), using $\mathrm{X}$-ray voxel Monte Carlo (XVMC), thereafter. Generally, gross tumor volume (GTV) was determined on CT images. Internal target volume (ITV) was created to cover GTV in all respiratory phases. Respiratory motion was assessed using CT and X-ray fluoroscopy. Slow scan CT was used before June 2009 and four-dimensional CT was used after that. A $5 \mathrm{~mm}$ margin was added to ITV to constitute planning target volume. Typically, five to eight non-coplanar static beams were arranged and irradiation was performed with $6 \mathrm{MV}$ photons. The dose was prescribed at the isocenter and dose prescription was determined according to tumor size and location: $48 \mathrm{~Gy}$ in four fractions for peripheral tumors (dose was escalated to $56 \mathrm{~Gy}$ in four fractions only for T2 tumors after June 2009), 6 0Gy in eight fractions for central tumors within $2 \mathrm{~cm}$ of the mediastinal structures (the trachea or proximal bronchial trees, great vessels, and heart), and 64 Gy in 16 fractions for central tumors with direct contact to mediastinal structures.

Follow-up schedule for the assessment of treatment. A follow-up chest CT was scheduled every 3 months for the first year and every 3-6 months thereafter. If chest CT findings or patient symptoms suggested disease progression, other appropriate examinations such as CT of other regions, PET-CT, or brain MRI were performed by treating physicians. Failure pattern was classified as local, regional, or distant recurrence and new primary lung cancer. Local recurrence was diagnosed by imaging findings (irradiated tumor size increase over 6 months or abnormal focal intense FDG uptake). Regional recurrence was defined as hilar, mediastinal, or supraclavicular lymph node metastases. Distant recurrence was defined as recurrence outside of the above-mentioned areas. New primary lung cancer was differentiated from pulmonary metastasis by a multidisciplinary tumor board, considering clinical factors such as time interval, morphologic feature, absence or presence of regional and distant metastasis, and histology if available.

Statistical analysis. Patients were divided into two groups according to CEA level: CEA-high patients and CEA-low patients. Various patient, tumor, and treatment factors were collected. A ground glass nodule (GGN) is a tumor composed of a solid portion with ground glass opacity. The cutoff value for CEA was set at the level of the upper normal limit $(5 \mathrm{ng} / \mathrm{ml})$. Patient characteristics were compared using Fisher's exact test for categorical variables and the Man-Whitney $U$ test for continuous variables. Spearman's rank correlation coefficient was used to determine the correlation between two variables.

Overall survival (OS) time was calculated from the initiation date of SBRT to the date of death and was censored at the date of the last follow-up contact for surviving patients. Progression-free survival (PFS) was calculated from the initiation date of SBRT to the date of death from any cause or recurrence, and was censored at the date of the last follow-up contact for surviving patients free from recurrence. OS and PFS times were estimated using the Kaplan-Meier method and compared by log-rank test. To examine the prognostic impact of CEA level on prognosis, univariate and multivariate analyses were performed using the Cox proportional hazards model. Factors with $p<0.1$ in univariate analysis were included in the multivariate analysis.

All statistical analyses were performed with EZR (Saitama Medical Center, Jichi Medical University, Saitama, Japan), a graphical user interface for R (The R Foundation for Statistical Computing, Vienna, Austria) (12). More precisely, EZR is a modified version of $\mathrm{R}$ Commander which provides statistical functions frequently used in biostatistics. $p$-Values were two-sided and a $p$-value $<0.05$ was regarded as statistically significant.

\section{Results}

Patient characteristics. A total of 195 patients with pathologically-confirmed stage I NSCLC received SBRT at our Department, and 129 of these patients were eligible for this retrospective study (exclusion reasons were as follows: synchronous or metachronous cancer $[\mathrm{n}=50]$, systemic chemotherapy before SBRT [ $\mathrm{n}=4]$, insufficient follow-up data $[\mathrm{n}=4]$, and absence of pretreatment CEA value $[\mathrm{n}=8]$ ). Of these 129 patients, 82 were classified as CEA-Low patients and 47 were classified as CEA-High patients. Patient characteristics are listed in Table I. There was no significant difference between CEA-Low and CEA-High patients in any characteristic except pretreatment CEA level, which was modestly elevated in CEAHigh patients (median=7.5, range $=5.1-50.7$ ). Clinical $\mathrm{T}$ stage appeared more advanced in CEA-High patients, but this observation was not statistically significant $(p=0.08)$ and the correlation between tumor size and CEA value was weak (Spearman's rank correlation coefficient of $0.21[p=0.02]$ ).

Survival and progression pattern. Median follow-up time was 38 months for all patients and 60 months for surviving patients. At the time of this analysis, 47 (57\%) patients in the CEA-Low group $(\mathrm{n}=87)$ and 29 patients (62\%) in the CEA-High group $(n=47)$ had deceased. Of 47 deaths in the CEA-Low group, 26 were attributed to lung cancer and 21 to other diseases. Of the 29 deaths in the CEA-High group, 22 were attributed to lung cancer and 7 to other diseases. Median OS time was 56 months (95\% CI=37-79) among CEA-Low patients and 43 months (95\%CI=24-76) among CEA-High patients $(p=0.39)$ (Figure $1 \mathrm{~A})$. The 3 -year OS rate was $63 \%(95 \% \mathrm{CI}=51-72)$ in CEALow patients and 57\% (95\% CI=41-70) in CEA-High patients. The cumulative incidence of lung cancer death tended to be worse in CEA-High patients, but this observation was not statistically significant $(p=0.07)$ (Figure $2 \mathrm{~A}$ ). The cumulative incidence of other causes of death was also not significantly different between the groups ( $p=0.20$ ) (Figure 2B). 
Table I. Patient characteristics.

\begin{tabular}{|c|c|c|c|}
\hline Characteristics & CEA-Low $(\mathrm{n}=82)$ & CEA-High $(\mathrm{n}=47)$ & $p$-Value \\
\hline Age (years), median (range) & $78(63-88)$ & $78(56-89)$ & 0.46 \\
\hline \multicolumn{4}{|l|}{ Gender, n (\%) } \\
\hline Male & $57(70)$ & $29(62)$ & 0.44 \\
\hline \multicolumn{4}{|l|}{ ECOG PS, n (\%) } \\
\hline $0-1$ & $72(88)$ & $43(91)$ & \multirow[t]{2}{*}{0.43} \\
\hline $2-3$ & $10(12)$ & $4(9)$ & \\
\hline \multicolumn{4}{|l|}{ Smoking history, n (\%) } \\
\hline Never & $18(22)$ & $10(21)$ & \multirow[t]{3}{*}{0.56} \\
\hline Current & $7(8)$ & $7(15)$ & \\
\hline Former & $57(70)$ & $30(64)$ & \\
\hline \multicolumn{4}{|l|}{ Operability, n (\%) } \\
\hline Yes & $31(38)$ & $15(32)$ & 0.57 \\
\hline \multicolumn{4}{|l|}{ Tumor location, n (\%) } \\
\hline Upper lobe & $51(62)$ & $23(51)$ & \multirow[t]{2}{*}{0.20} \\
\hline Middle/Lower lobe & $31(38)$ & $24(49)$ & \\
\hline \multicolumn{4}{|l|}{ Clinical T stage, $\mathrm{n}(\%)$} \\
\hline $1 \mathrm{a} / 1 \mathrm{~b} / 2 \mathrm{a}$ & $41(50) / 25(30) / 16(20)$ & $14(30) / 20(42) / 13(28)$ & 0.08 \\
\hline GGN, n (\%) & $10(12)$ & $3(6)$ & 0.37 \\
\hline Staging with PET, n (\%) & $74(90)$ & $40(85)$ & 0.40 \\
\hline CEA (ng/ml), median (range) & $2.9(0.1-4.8)$ & $7.5(5.1-50.7)$ & $<0.001$ \\
\hline CYFRA (ng/ml), median (range) & $2.45(0.9-10.1)$ & $2.95(0.7-6.8)$ & 0.08 \\
\hline $\mathrm{NA}, \mathrm{n}(\%)$ & $4(5 \%)$ & $1(2 \%)$ & \\
\hline $\mathrm{SCC}(\mathrm{mg} / \mathrm{mL})$, median (range) & $1.3(0.4-26.6)$ & $1.2(0.4-6.7)$ & 0.85 \\
\hline $\mathrm{NA}, \mathrm{n}(\%)$ & $10(12 \%)$ & $6(13 \%)$ & \\
\hline \multicolumn{4}{|l|}{ Histology, n (\%) } \\
\hline Adenocarcinoma & $41(50)$ & $23(49)$ & \multirow[t]{4}{*}{0.47} \\
\hline Squamous cell & $34(40)$ & $16(34)$ & \\
\hline Large cell & $1(1)$ & $1(2)$ & \\
\hline NSCLC, NOS & $6(7)$ & $7(15)$ & \\
\hline \multicolumn{4}{|l|}{ Dose prescription, n (\%) } \\
\hline $48 \mathrm{~Gy} / 4 \mathrm{fr}$ & $65(79)$ & $33(70)$ & \multirow[t]{4}{*}{0.28} \\
\hline $56 \mathrm{~Gy} / 4 \mathrm{fr}$ & $6(7)$ & $4(9)$ & \\
\hline $60 \mathrm{~Gy} / 8 \mathrm{fr}$ & $11(14)$ & $8(17)$ & \\
\hline $64 \mathrm{~Gy} / 16 \mathrm{fr}$ & $0(0)$ & $2(4)$ & \\
\hline
\end{tabular}

CEA: Carcinoembryonic antigen; ECOG: Eastern Cooperative Oncology Group; PS: performance status, PET: positron emission tomography; NSCLC: non-small cell lung cancer; NOS: Not otherwise specified; fr: fraction; GGN: ground glass nodule.

At the time of this analysis, the number of events in PFS (death or disease progression) was $52(63 \%)$ among CEALow patients $(\mathrm{n}=82)$ and $36(77 \%)$ among CEA-High patients $(\mathrm{n}=47)$. The median PFS time was 38 months (95\% CI=24-58) in CEA-Low patients versus 16 months (95\% CI $=12-27)$ in CEA-High patients $(p=0.01)$ (Figure 1B). The 3-year PFS rate in CEA-Low patients and CEA-High patients was $51 \%(95 \% \mathrm{CI}=29-61)$ and $31 \%(95 \% \mathrm{CI}=18-45)$, respectively.

The failure pattern details are listed in Table II. Crude rates of local, regional, distant metastasis, and new primary lung cancer were $22 \%, 9 \%, 20 \%$, and $4 \%$, respectively, in CEA-Low patients versus $26 \%, 23 \%, 38 \%$, and $0 \%$ in CEAHigh patients. Thus, recurrence in regional nodes and distant sites was more common in CEA-High patients.
Prognostic factor and the effect of histology on the prognostic impact of CEA level. Univariate and multivariate analysis for PFS is shown in Table III. In univariate analysis, age (>75), CEA, tumor size, and GGN were significant factors. In multivariate analysis, high CEA level and larger tumor size remained independent prognostic factors for worse PFS (HR, 1.67 [95\%CI=1.07-2.62] and 1.42 [95\% CI=1.08-1.87], respectively).

To determine the effect of histology on the prognostic impact of CEA, Kaplan-Meier curves of PFS according to histology (adenocarcinoma or non-adenocarcinoma) are shown in Figure 3. For adenocarcinoma patients, PFS in CEA-High patients was significantly worse than that in CEA-Low patients (3-year PFS rate, 26\% [95\%CI=11-45] vs. $57 \%$ [95\% CI=40-71]; $p=0.01$ ) (Figure 3A). No significant 
$\mathbf{A}$

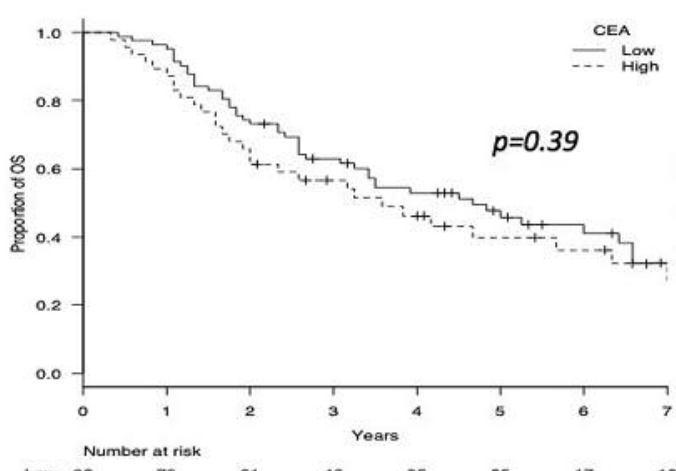

B

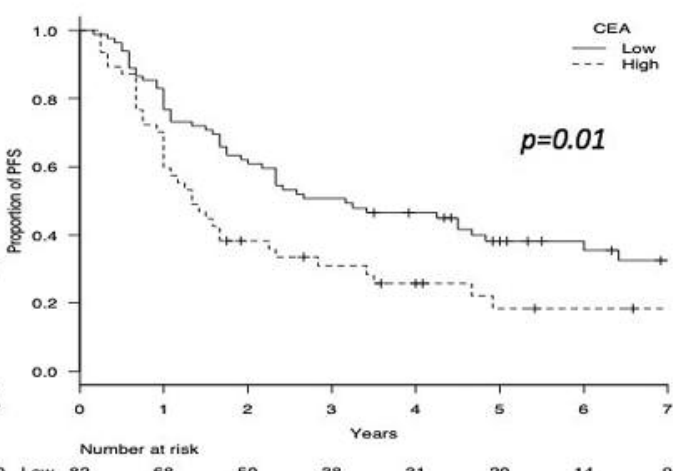

Figure 1. Kaplan-Meier curves of overall survival (OS) (A) and progression-free survival (PFS) (B) according to pretreatment CEA value. CEA: Carcinoembryonic antigen.

$\mathbf{A}$

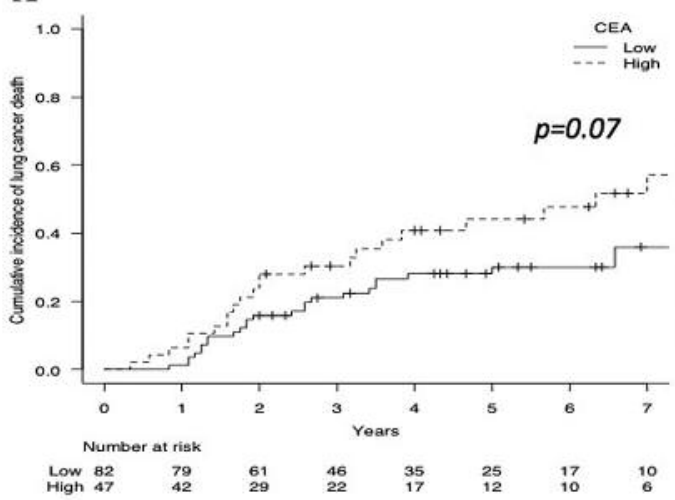

B

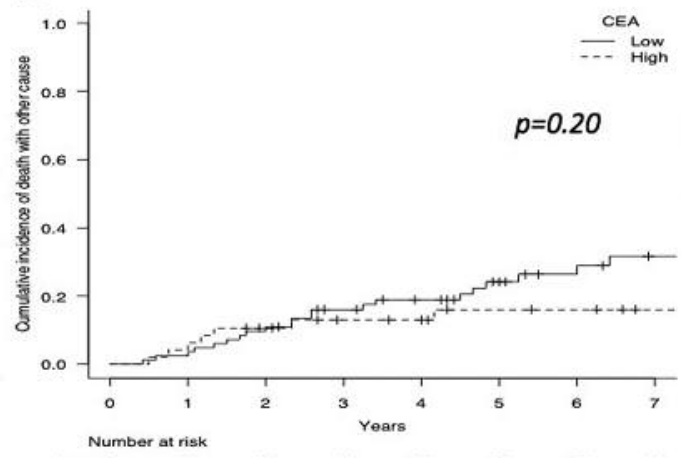

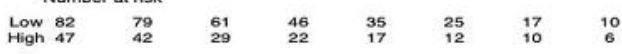

Figure 2. Cumulative incidence of lung cancer death (A) and other cause of death (B) according to pretreatment CEA value. CEA: Carcinoembryonic antigen.

A

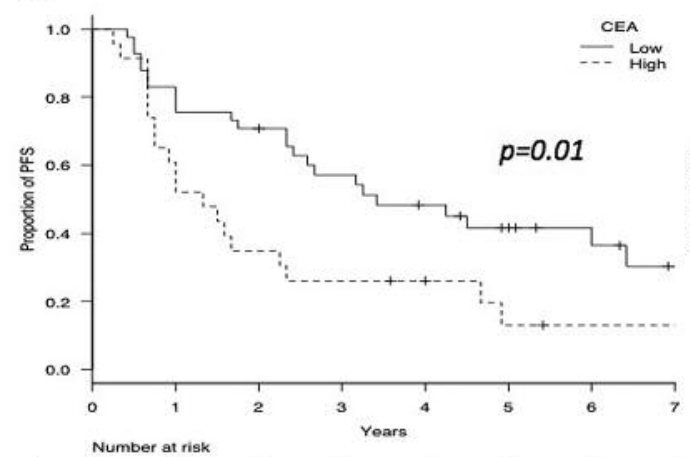

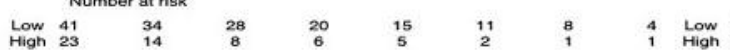

B

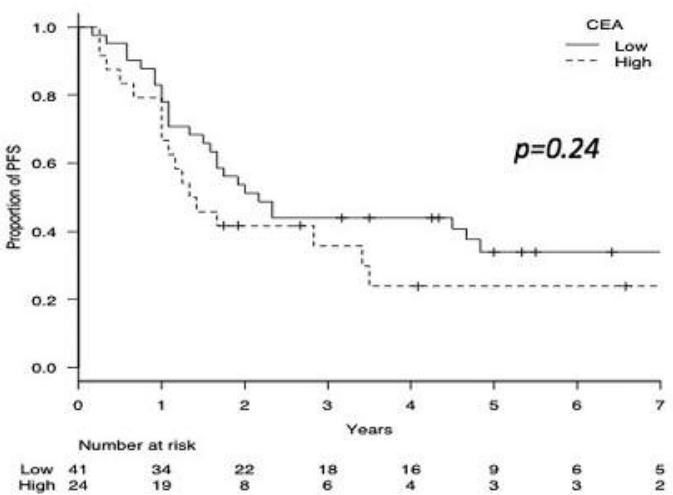

Figure 3. Kaplan-Meier curves of progression-free survival in adenocarcinoma (A) and non-adenocarcinoma patients according to pretreatment CEA value. CEA: Carcinoembryonic antigen. 
Table II. Patterns of treatment failure.

\begin{tabular}{lcc}
\hline & CEA-Low $(\mathrm{n}=82)$ & CEA-High $(\mathrm{n}=47)$ \\
\hline Local (L) & $18(22 \%)$ & $12(26 \%)$ \\
L only & 9 & 7 \\
L and R & 2 & 2 \\
L and D & 5 & 1 \\
L, R and D & 2 & 2 \\
Regional node (R) & $7(9 \%)$ & $11(23 \%)$ \\
R only & 3 & 3 \\
R and D & 0 & 4 \\
Distant (D) & $17(20 \%)$ & $18(38 \%)$ \\
D only & 10 & 11 \\
New primary lung cancer & $3(4 \%)$ & 0 \\
\hline
\end{tabular}

CEA: Carcinoembryonic antigen.

difference was observed in the subset of nonadenocarcinoma patients (3-year PFS rate, 36\% [95\% CI=1755] vs. $44 \%$ [95\% CI=29-58]; $p=0.24)$ (Figure 3B).

\section{Discussion}

In this study, we showed that CEA-High stage I NSCLC patients who received SBRT had poorer PFS with higher rates of regional and distant metastasis, although the difference in OS was not significant.

PET is expected to improve the accuracy of staging, although the upstaging rate in early-stage lung cancer is not high (7\% according to the subset analysis of clinical stage IA NSCLC patients in the ACOSOG Z0050 trial) (13). According to a meta-analysis of PET for clinical stage T12N0 NSCLC, the negative predictive value for nodal staging was $94 \%$ for $\mathrm{T} 1$ tumors and $89 \%$ for $\mathrm{T} 2$ tumors (corresponding to $6 \%$ and $11 \%$ occult disease, respectively) (14). In fact, regional node recurrence rate was approximately $10 \%$ after lung SBRT, as reported by Chi et al. (1). Some retrospective studies comparing clinical stage with pathologic stage reported potential risk factors for occult nodal metastasis, such as centrally located tumor and larger tumor (T2 disease) $(15,16)$. Most of our cohorts were staged with PET-CT (approximately 90\%) and had small tumors (clinical T1 disease; approximately $80 \%$ ). In addition, most patients (approximately 80\%) were treated with regimens for peripherally located tumors. Nevertheless, the rate of regional failure was particularly high $(23 \%)$ in CEA-High patients in our study. According to the largest retrospective study of clinical stage I NSCLC $(n=1000)$ patients staged without PET-CT and treated with surgery, pathologic regional node metastasis was more common in CEA-High patients $(29 \%)$ than that in CEA-Low patients (17\%) and the increased risk of systemic micrometastasis was suggested by the finding that
Table III. Univariate and multivariate analysis for progression-free survival.

\begin{tabular}{lccc}
\hline & HR & $95 \%$ CI & $p$-Value \\
\hline Univariate analysis & & & \\
Age $(>75)$ & 1.68 & $1.05-2.69$ & 0.032 \\
CEA ( $>5$ ng/ml) & 1.73 & $1.12-2.67$ & 0.013 \\
Tumor size (per 1 cm) & 1.61 & $1.24-2.09$ & $<0.001$ \\
GGN & 0.21 & $0.05-0.86$ & 0.030 \\
Histology (non-adeno $v s$. adeno) & 0.96 & $0.63-1.46$ & 0.83 \\
Operability (Yes $v s$. No) & 0.86 & $0.56-1.35$ & 0.52 \\
PS (2-3 vs. 0-1) & 1.37 & $0.71-2.66$ & 0.35 \\
Male & 1.19 & $0.75-1.88$ & 0.45 \\
Smoking history & & & \\
$\quad$ Current or Former $v s$. Never) & 1.21 & $0.71-2.01$ & 0.49 \\
Fraction $(8$ or 16 fr $v s .4$ fr) & 1.23 & $0.70-2.16$ & 0.47 \\
SCC (High; $>1.5)$ & 1.31 & $0.82-2.09$ & 0.25 \\
CYFRA (High; $>2.2)$ & 1.08 & $0.70-1.66$ & 0.73 \\
Multivariate analysis & & & \\
CEA (>5 ng/ml) & 1.67 & $1.07-2.62$ & 0.025 \\
Tumor size (per 1 cm) & 1.42 & $1.08-1.87$ & 0.012 \\
Age ( $>75)$ & 1.47 & $0.89-2.42$ & 0.13 \\
GGN & 0.25 & $0.06-1.01$ & 0.052 \\
\hline
\end{tabular}

CEA: Carcinoembryonic antigen; GGN: ground glass nodule; adeno: adenocarcinoma; PS: performance status; fr: fraction.

CEA was a negative prognostic factor also for pathological stage I patients (8). Shingyoji et al. reported that CEA significantly correlated with pathologic N2 disease in clinical NO patients staged even with the use of PET-CT (17). Thus, CEA-High stage I NSCLC patients had higher risk of micrometastases in regional node and distant site, even when staged with PET-CT.

A number of surgical series has addressed the prognostic impact of CEA and the majority of the studies reported that high CEA was a negative prognostic factor for OS while a minority did not support this finding $(8,18,19)$. We observed no significant difference in OS despite a marked difference in PFS in this study. This was probably caused by various factors, such as salvage therapy after recurrence, lack of sample size and, especially, a high rate of other causes of death. High proportions of other causes of death are often observed in patients treated with SBRT because these patients are generally older and have other medical comorbidities. In our cohorts, the proportion of other causes of death in all deaths was $44 \%$ in CEA-Low patients versus $24 \%$ in CEA-High patients. When considering other causes of death as a competing risk, the cumulative incidence of lung cancer deaths appeared higher in CEA-High patients, although this observation was not statistically significant. Therefore, we cannot exclude the possibility that CEA is a risk factor for lung cancer death, and studies of larger cohorts are needed to draw a firm conclusion. 
CEA is a more sensitive and specific marker for adenocarcinoma than for squamous cell carcinoma, and whether it is a prognostic factor for non-adenocarcinoma remains undetermined. Some surgical series have reported that CEA is a poor prognostic factor for squamous cell carcinoma (20) while others suggest contradictorily $(19,21$, 22). When we compared PFS according to histology, CEAHigh adenocarcinoma patients had poorer PFS than CEALow patients. In contrast, no notable difference was observed between non-adenocarcinoma patients according to CEA level. Although each subset of patients classified according to histology was small, the prognostic impact of pretreatment CEA level could be important, especially for adenocarcinoma patients.

There were several limitations to this study. First, the study was retrospective in design and pretreatment CEA levels were not available for all stage I NSCLC patients. However, only eight patients were excluded through lack of pretreatment CEA, and we consider that the study cohorts were representative of stage I NSCLC patients. Second, other tumor marker levels (CYFRA and SCC) were not available for all patients. Again, the number of patients without these data was very small and the effect on study outcome was likely minimal, if any. Third, staging PET was performed at the various institutions using different image acquisition protocols and thus we did not assess the prognostic impact of $\mathrm{SUV}_{\max }$ in this study, although this parameter has been reported as a prognostic factor in some studies $(23,24)$. Last, we were unable to examine the prognostic impact of posttreatment CEA level because of the small number of patients with elevated pretreatment CEA $(n=47)$ and the inherent timing of CEA measurement after SBRT. Modest elevation of CEA is often observed in benign conditions such as advanced age, smoking, liver damage, renal failure, diabetes mellitus, and inflammatory disease, conditions which are often associated with patients receiving SBRT (7), and we therefore could not determine whether an elevated CEA level was the result of lung cancer or had another cause at the time of diagnosis. A surgical paper has reported the usefulness of postsurgical CEA level for pretreatment of CEA-High patients (8). Thus, posttreatment CEA value may have more important prognostic significance than pretreatment CEA for patients receiving SBRT, and further studies are warranted to address this question.

In conclusion, elevated CEA and larger tumor size were independent prognostic factors for worse PFS and failure pattern analysis showed that the risk of occult micrometastases in regional nodes and distant sites was higher in CEA-High patients, even when PET-CT was used for staging. Therefore, stage I NSCLC patients with high CEA, especially those with adenocarcinoma, should be followed-up carefully after SBRT.

\section{Acknowledgements}

This study was supported by the Japan Society for the Promotion of Science Grant-in-Aid for Scientific Research (C) 15K09992. The study sponsor had no involvement in the study design and the collection, analysis, and interpretation of data; and in the decision to submit the manuscript for publication.

\section{Conflicts of Interest}

None.

\section{References}

1 Chi A, Liao Z, Nguyen NP, Xu J, Stea B and Komaki R: Systemic review of the patterns of failure following stereotactic body radiation therapy in early-stage non-small-cell lung cancer: clinical implications. Radiother Oncol 94: 1-11, 2010.

2 Janssen S, Kasmann L, Rudat V and Rades D: Stereotactic Body Radiation Therapy (SBRT) for Recurrent Non-small Cell Lung Cancer (NSCLC). Anticancer Res 36: 825-828, 2016.

3 Timmerman R, Paulus R, Galvin J, Michalski J, Straube W, Bradley J, Fakiris A, Bezjak A, Videtic G, Johnstone D, Fowler J, Gore E and Choy H: Stereotactic body radiation therapy for inoperable early stage lung cancer. JAMA 303: 1070-1076, 2010.

4 Nagata Y, Hiraoka M, Shibata T, Onishi H, Kokubo M, Karasawa K, Shioyama Y, Onimaru R, Kozuka T, Kunieda E, Saito T, Nakagawa K, Hareyama M, Takai Y, Hayakawa K, Mitsuhashi N and Ishikura S: Prospective Trial of Stereotactic Body Radiation Therapy for Both Operable and Inoperable T1N0M0 Non-Small Cell Lung Cancer: Japan Clinical Oncology Group Study JCOG0403. Int J Radiat Oncol Biol Phys 93: 989996, 2015.

5 Chang JY, Senan S, Paul MA, Mehran RJ, Louie AV, Balter P, Groen HJM, McRae SE, Widder J, Feng L, van den Borne BEEM, Munsell MF, Hurkmans C, Berry DA, van Werkhoven E, Kresl JJ, Dingemans A-M, Dawood O, Haasbeek CJA, Carpenter LS, De Jaeger K, Komaki R, Slotman BJ, Smit EF and Roth JA: Stereotactic ablative radiotherapy versus lobectomy for operable stage I non-small-cell lung cancer: a pooled analysis of two randomised trials. Lancet Oncol 16: 630-637, 2015.

6 Onishi H, Shirato H, Nagata Y, Hiraoka M, Fujino M, Gomi K, Karasawa K, Hayakawa K, Niibe Y, Takai Y, Kimura T, Takeda A, Ouchi A, Hareyama M, Kokubo M, Kozuka T, Arimoto T, Hara R, Itami J and Araki T: Stereotactic body radiotherapy (SBRT) for operable stage I non-small-cell lung cancer: can SBRT be comparable to surgery? Int J Radiat Oncol Biol Phys 81: 1352-1358, 2011.

7 Okamura K, Takayama K, Izumi M, Harada T, Furuyama K and Nakanishi Y: Diagnostic value of CEA and CYFRA 21-1 tumor markers in primary lung cancer. Lung Cancer 80: 45-49, 2013.

8 Okada M, Nishio W, Sakamoto T, Uchino K, Yuki T, Nakagawa A and Tsubota N: Prognostic significance of perioperative serum carcinoembryonic antigen in non-small cell lung cancer: analysis of 1,000 consecutive resections for clinical stage I disease. Ann Thorac Surg 78: 216-221, 2004.

9 Matsuo Y, Shibuya K, Nagata Y, Takayama K, Norihisa Y, Mizowaki T, Narabayashi M, Sakanaka $\mathrm{K}$ and Hiraoka M: Prognostic factors in stereotactic body radiotherapy for non-smallcell lung cancer. Int J Radiat Oncol Biol Phys 79: 1104-1111, 2011. 
10 Tsurugai $\mathrm{Y}$, Kozuka T, Ishizuka N and Oguchi M: Relationship between the consolidation to maximum tumor diameter ratio and outcomes following stereotactic body radiotherapy for stage I non-small-cell lung cancer. Lung Cancer 92: 47-52, 2016.

11 Takayama K, Nagata Y, Negoro Y, Mizowaki T, Sakamoto T, Sakamoto M, Aoki T, Yano S, Koga S and Hiraoka M: Treatment planning of stereotactic radiotherapy for solitary lung tumor. Int J Radiat Oncol Biol Phys 61: 1565-1571, 2005.

12 Kanda Y: Investigation of the freely available easy-to-use software 'EZR' for medical statistics. Bone Marrow Transplant 48: 452-458, 2013.

13 Kozower BD, Meyers BF, Reed CE, Jones DR, Decker PA and Putnam JB Jr:: Does positron emission tomography prevent nontherapeutic pulmonary resections for clinical stage IA lung cancer? Ann Thorac Surg 85: 1166-1169, 2008.

14 Wang J, Welch K, Wang L and Kong FM: Negative predictive value of positron emission tomography and computed tomography for stage T1-2N0 non-small-cell lung cancer: a meta-analysis. Clin Lung Cancer 13: 81-89, 2012.

15 Lee PC, Port JL, Korst RJ, Liss Y, Meherally DN and Altorki NK: Risk factors for occult mediastinal metastases in clinical stage I non-small cell lung cancer. Ann Thorac Surg 84: 177$181,2007$.

16 Gomez-Caro A, Boada M, Cabanas M, Sanchez M, Arguis P, Lomena F, Ramirez J and Molins L: False-negative rate after positron emission tomography/computer tomography scan for mediastinal staging in cI stage non-small-cell lung cancer. Eur J Cardiothorac Surg 42: 93-100, 2012.

17 Shingyoji M, Nakajima T, Yoshino M, Yoshida Y, Ashinuma H, Itakura $\mathrm{M}$, Tatsumi $\mathrm{K}$ and Iizasa $\mathrm{T}$ : Endobronchial ultrasonography for positron emission tomography and computed tomography-negative lymph node staging in non-small cell lung cancer. Ann Thorac Surg 98: 1762-1767, 2014.

18 Matsuguma H, Nakahara R, Igarashi S, Ishikawa Y, Suzuki H, Miyazawa N, Honjo S and Yokoi K: Pathologic stage I nonsmall cell lung cancer with high levels of preoperative serum carcinoembryonic antigen: clinicopathologic characteristics and prognosis. J Thorac Cardiovasc Surg 135: 44-49, 2008.
19 Kato T, Ishikawa K, Aragaki M, Sato M, Okamoto K, Ishibashi T, Oba K and Kaji M: Optimal predictive value of preoperative serum carcinoembryonic antigen for surgical outcomes in stage I non-small cell lung cancer: differences according to histology and smoking status. J Surg Oncol 107: 619-624, 2013.

20 Nagashima T, Sakao Y, Mun M, Ishikawa Y, Nakagawa K, Masuda M and Okumura S: A clinicopathological study of resected small-sized squamous cell carcinomas of the peripheral lung: prognostic significance of serum carcinoembryonic antigen levels. Ann Thorac Cardiovasc Surg 19: 351-357, 2013.

21 Okada M, Nishio W, Sakamoto T, Uchino K, Yuki T, Nakagawa A and Tsubota N: Effect of histologic type and smoking status on interpretation of serum carcinoembryonic antigen value in non-small cell lung carcinoma. Ann Thorac Surg 78: 1004-1009, 2004.

22 Tomita M, Matsuzaki Y, Edagawa M, Shimizu T, Hara M and Onitsuka T: Prognostic significance of preoperative serum carcinoembryonic antigen level in lung adenocarcinoma but not squamous cell carcinoma. Ann Thorac Cardiovasc Surg 10: 7680, 2004.

23 Takeda A, Sanuki N, Fujii H, Yokosuka N, Nishimura S, Aoki Y, Oku Y, Ozawa Y and Kunieda E: Maximum standardized uptake value on FDG-PET is a strong predictor of overall and disease-free survival for non-small-cell lung cancer patients after stereotactic body radiotherapy. J Thorac Oncol 9: 65-73, 2014.

24 Kohutek ZA, Wu AJ, Zhang Z, Foster A, Din SU, Yorke ED, Downey R, Rosenzweig KE, Weber WA and Rimner A: FDGPET maximum standardized uptake value is prognostic for recurrence and survival after stereotactic body radiotherapy for non-small cell lung cancer. Lung Cancer 89: 115-120, 2015.

Received June 30, 2017

Revised July 8, 2017

Accepted July 10, 2017 